\title{
Divergência genética entre clones de guaranazeiro(1)
}

\author{
Firmino José do Nascimento Filho(2), André Luiz Atroch ${ }^{(2)}$, Nelcimar Reis de Sousa ${ }^{(2)}$,
} Terezinha Batista Garcia(2), Manoel da Silva Cravo(2) e Enilton Fick Coutinho( ${ }^{(2)}$

Resumo - As técnicas multivariadas, para estimar a diversidade genética de um grupo de progenitores, têm sido utilizadas com freqüência pelos melhoristas de plantas. Os progenitores são utilizados em cruzamentos biparentais ou múltiplos, para formação de populações segregantes que tenham maior probabilidade de recuperação de genótipos superiores. Este trabalho foi realizado com o objetivo de identificar clones de guaranazeiro produtivos e divergentes que possam ser utilizados em um programa de cruzamentos para obter híbridos com alto valor heterótico e materiais para propagação vegetativa. Foram avaliados 148 clones de guaranazeiro atualmente em uso no programa de melhoramento genético da Embrapa-Centro de Pesquisa Agroflorestal da Amazônia Ocidental. Utilizou-se, para estimativa da divergência genética, a análise de agrupamento, em que a medida de dissimilaridade utilizada foi a distância euclidiana média padronizada e os métodos de agrupamento de otimização de Tocher e do vizinho mais próximo para construção do dendrograma entre grupos de clones. Houve a formação de sete grupos divergentes de clones. Concluiu-se que a divergência genética entre os clones não é grande, pois dois grupos foram formados com dois clones e três grupos foram formados somente com um único clone. Os clones CMU384 e CMU801 foram os mais próximos geneticamente e podem ser utilizados na formação de uma população com desenvolvimento vegetativo uniforme para uso em plantios comerciais.

Termos para indexação: Paullinia cupana, distância genética, cruzamento, híbridos, população de plantas, métodos de melhoramento.

\section{Genetic divergence between clones of guarana}

Abstract - The multivariate techniques to estimate the genetic diversity of a group of progenitors has been used by plant breeders. The progenitors are still being used in parental or multiple crossings to form segregating populations that have larger probability of recovering superior genotypes. This work was carried out with the objective of identifying guarana clones with high production and divergent clones that can be used in a crossing program in order to obtain hybrids with heterosis, as well as materials for vegetative propagation. One hundred and forty-eight clones of guarana in use at this moment in the genetic breeding program of Embrapa-Centro de Pesquisa Agroflorestal da Amazônia Ocidental were studied. To estimate the genetic divergence, the cluster analysis was used in which the measure of dissimilarity used was the euclidian average standardized distance and the Tocher's method of grouping and a single linkage method to make up a dendrogram between groups of clones. There was formation of seven divergent groups. It was concluded that the genetic divergence between the clones is not large because two groups with two clones and three groups with one clone have been formed. The CMU384 and CMU801 clones were the genetically closest and could be used to form a population with uniform vegetative development for use in commercial plantations.

Index terms: Paullinia cupana, genetic distance, crossbreeding, hybrids, plant population, breeding methods.

(1) Aceito para publicação em 21 de junho de 2000 .

(2) Embrapa-Centro de Pesquisa Agroflorestal da Amazônia Ocidental, Caixa Postal 319, CEP 69011-970 Manaus, AM.

E-mail: atroch@cpaa.embrapa.br, firmino@cpaa.embrapa.br, nelcimar@cpaa.embrapa.br, tgarcia@cpaa.embrapa.br, coutinho@cpaa.embrapa.br

\section{Introdução}

O conhecimento da diversidade genética entre um grupo de progenitores é de grande importância para qualquer programa de melhoramento genético. Isto porque é necessário identificar combinações híbri- 
das de maior efeito heterótico, em cujas descendências tenha-se maior probabilidade de recuperação de genótipos superiores (Cruz \& Regazzi, 1997). Além disso, as medidas de distância genética têm sido úteis na avaliação de acessos em bancos de germoplasma, no estabelecimento das relações entre a diversidade genética e geográfica, e também para evitar a vulnerabilidade genética das culturas.

A utilização de técnicas multivariadas para estimar a divergência genética tem-se tornado comum entre os melhoristas de plantas. Entre essas práticas, as mais empregadas são: a análise por componentes principais, quando os dados são obtidos de experimentos sem repetições; a análise por variáveis canônicas, quando os dados são obtidos de experimentos com repetições; e, por último, os métodos de agrupamento, cuja aplicação depende da utilização de uma medida de dissimilaridade previamente estimada (Machado, 1999).

Entre as medidas de dissimilaridade, a distância euclidiana e a distância $\mathrm{D}^{2}$ de Mahalanobis são as mais utilizadas nos programas de melhoramento genético. Um dos inconvenientes apresentados pela distância euclidiana é o fato de ela ser alterada com a mudança de escala de medições, com o número de caracteres estudados, e de não levar em conta o grau de correlação entre eles. No entanto, para contornar o problema de escala, tem sido recomendada a padronização dos dados, e para contornar a influência do número de caracteres, utilizou-se a distância euclidiana média (Cruz \& Regazzi, 1997).

A análise de agrupamento, segundo Cruz \& Regazzi (1997), tem por finalidade reunir, por algum critério de classificação, os progenitores (ou qualquer outro tipo de unidade amostral) em vários grupos, de tal forma que exista homogeneidade dentro do grupo e heterogeneidade entre grupos.

Com a possibilidade da clonagem pelo método da estaquia, a estratégia de melhoramento genético utilizada na cultura do guaraná tem sido a seleção clonal, ou seja, plantas superiores de diversas populações são propagadas assexuadamente. Estes clones são avaliados em ensaios de competição, e os melhores são recomendados para plantio comercial. Entretanto, esta estratégia pode ter como conseqüência a limitação da diversidade genética, a suscetibilidade dos genótipos a fatores bióticos e abióticos em face da uniformidade do plantio e a redução da possibilidade de ganhos adicionais futuros nos programas de seleção, uma vez que o melhorista passa a manejar um "pool" gênico de tamanho limitado. Duas alternativas são viáveis para ampliar a variabilidade genética no guaraná: uso da seleção recorrente e hibridação, visando à obtenção de variedades ou híbridos superiores.

Em um programa de hibridação, a escolha dos progenitores é o passo fundamental para o sucesso do programa. Estes progenitores devem apresentar bom desempenho e grande divergência genética, sob o risco de não se ampliar variabilidade genética suficiente para se obterem ganhos com a seleção.

O presente trabalho teve como objetivo identificar clones que possam ser utilizados em um programa de cruzamentos biparentais ou cruzamentos múltiplos, visando à obtenção de híbridos com alto valor heterótico e de materiais para propagação vegetativa.

\section{Material e Métodos}

O trabalho foi realizado na Embrapa-Centro de Pesquisa Agroflorestal da Amazônia Ocidental, em Manaus, AM.

Os dados foram coletados em 148 clones de guaranazeiro (Paullinia cupana var. sorbilis), em experimentos de competição de clones, e no Banco Ativo de Germoplasma, instalados no Campo Experimental de Manaus, localizado a uma latitude de $3^{\circ} 8^{\prime} 5^{\prime \prime} \mathrm{S}$, longitude de $60^{\circ} 1^{\prime} \mathrm{W}$ de GRT e numa altitude de $50 \mathrm{~m}$ acima do nível do mar; tipo climático Afi da classificação de Köppen (clima tropical chuvoso), caracterizado por apresentar temperatura média do mês mais frio nunca inferior a $18^{\circ} \mathrm{C}$, e a precipitação pluvial do mês mais seco, acima de $60 \mathrm{~mm}$ (Boletim Agrometeorológico, 1998)

As características utilizadas para o cálculo da divergência genética foram o comprimento do ramo principal aos 12 meses de idade (CRP), o número de ramos aos 12 meses de idade (NR), o número de folhas aos 12 meses de idade (NF) e a produção de sementes torradas (PROD), em kg/planta (média de seis anos de avaliação).

A divergência genética foi estimada por análise de agrupamento, em que a medida de dissimilaridade utilizada foi a distância euclidiana média padronizada, e o método de agrupamento, o de otimização de Tocher. A formação dos grupos teve como critério o valor máximo da medida de dissimilaridade encontrado no conjunto das menores distâncias envolvendo cada progenitor

A padronização dos dados foi realizada de acordo com Cruz \& Regazzi (1997), por: 
$\mathrm{x}_{\mathrm{ij}}=\frac{\mathrm{X}_{\mathrm{ij}}}{\mathrm{S}\left(\mathrm{X}_{\mathrm{j}}\right)}$

em que $S\left(X_{j}\right)$ é o desvio-padrão dos dados do j-ésimo caráter; então,

$\mathrm{d}_{\mathrm{ii}}=\sqrt{1 / \mathrm{n} \sum_{\mathrm{j}}\left(\mathrm{x}_{\mathrm{ij}}-\mathrm{x}_{\mathrm{i}^{\mathrm{ij}}}\right)^{2}}$

é a distância euclidiana média baseada em dados padronizados, em que $n$ é o número de caracteres analisados, e $\mathrm{x}_{\mathrm{ij}}$ é a observação no i-ésimo progenitor ( $i=1,2, \ldots$ p), em referência ao j-ésimo caráter $(\mathrm{j}=1,2, \ldots \mathrm{n})$ estudado.

Foi utilizado o programa GENES (Cruz, 1997) para as análises de divergência genética.

Após a formação inicial dos grupos, realizou-se a análise de variância entre e dentro de grupos, em relação a cada característica, utilizando o procedimento General Linear Model (GLM) do programa SAS.

Também utilizou-se o método do vizinho mais próximo para formação de um dendrograma entre os grupos de clones, por meio do programa MAPGEN, da Universidade Federal de Lavras (Minas Gerais).

\section{Resultados e Discussão}

Os clones avaliados, bem como as médias originais relativas aos caracteres CRP, NR, NF e PROD, encontram-se na Tabela 1.

A Tabela 2 resume os resultados das estimativas das distâncias genéticas dos 148 clones. Os clones mais distantes geneticamente foram CMA247 e CMU687, e os mais próximos, CMU384 e CMU801.

As estimativas das distâncias genéticas permitiram a formação de sete grupos distintos, pelo método de Tocher (Tabela 3). Este resultado indica que a divergência genética entre os clones, atualmente em uso no programa de melhoramento genético do guaranazeiro da Embrapa-Centro de Pesquisa Agroflorestal da Amazônia Ocidental, não é grande, pois dois grupos foram formados com dois clones, e três grupos foram formados somente com um único clone, concentrando-se a maioria dos clones em dois grupos.

Isto pode ser explicado pelo fato de que as coletas de ramos de matrizes para formação dos clones foi restrita aos municípios de Manaus, Maués e Iranduba, no Estado do Amazonas, que se mostraram insuficientes para representar a diversidade genética da cultura. Ou, que a diversidade geográfica não está correlacionada com a diversidade genética, fato confirmado pela existência de clones de origens geográficas diferentes, que foram classificados no mesmo grupo de divergência genética, e clones da mesma origem geográfica classificados em grupos diferentes.

Cruz (1990) relata que a escolha de progenitores para cruzamentos tem sido feita, algumas vezes, tomando-se a diversidade geográfica como indicador da diversidade genética. Porém este critério tem recebido críticas, pelo fato de não quantificar a divergência entre as populações, e, em muitos casos, por não existir relação entre a divergência genética e a diversidade geográfica.

Murty \& Arunachalam (1966) e Upadhyay \& Murty (1970) citam que a deriva genética e a seleção em vários ambientes podem causar maior diversidade que a distância geográfica. Além disso, atualmente, as trocas de germoplasma de várias espécies entre pesquisadores e instituições causam perdas de individualidade e ocorrência de tipos particulares nas regiões, em virtude da interferência humana (Cruz, 1990).

A origem e evolução do guaranazeiro, bem como sua domesticação pelos índios amazônidas, e a seleção artificial realizada por instituições de pesquisa, corroboram os resultados obtidos neste trabalho.

Conforme Ducke (1937), a cultura do guaranazeiro propagou-se das suas origens do alto Orenoco e alto Rio Negro venezuelano, para o baixo Rio Negro, onde está estabelecida a sua maior área de cultivo, a região de Maués no Amazonas, delimitada pelos rios Madeira, Maués e Paraná dos Ramos. Entretanto, Cavalcante (1967) é da opinião de que o possível centro de origem do guaranazeiro seria o $\mathrm{Mu}$ nicípio de Santarém, Pará, por ter sido encontrado em estado provavelmente espontâneo em uma mata virgem da região.

A estimativa da divergência genética na cultura do guaranazeiro apresenta duas vantagens, segundo Nascimento Filho et al. (1992): a identificação de progenitores com máxima divergência genética, destinados a cruzamentos; e a identificação de progenitores produtivos com máxima similaridade genética, destinados à propagação vegetativa.

Os resultados obtidos indicam que, para se obterem populações segregantes com possibilidades de superioridade sobre os pais a partir de cruzamentos biparentais, os clones mais produtivos de cada grupo deveriam ser intercruzados. Entretanto, o fato de dois genitores serem divergentes não implica supe- 
Tabela 1. Médias de comprimento do ramo principal (CRP), em cm, número de ramos (NR) e número de folhas (NF), aos 12 meses de idade, e produção de sementes torradas (PROD), em kg/planta, de 148 clones de guaranazeiro.

\begin{tabular}{|c|c|c|c|c|c|c|c|c|c|c|c|c|c|c|}
\hline Clone $^{(1)}$ & CPR & NR & $\mathrm{NF}$ & PROD & Clone & CPR & $\mathrm{NR}$ & $\mathrm{NF}$ & PROD & Clone & CPR & NR & NF & PROD \\
\hline CMA183 & 93,80 & 9,10 & 24,40 & 0,60 & CMU259 & 80,60 & 8.90 & 30,90 & 0,55 & CMU625 & $\begin{array}{l}96,40 \\
\end{array}$ & 6.40 & $\begin{array}{l}21,90 \\
\end{array}$ & 0,74 \\
\hline CMA1818 & 64.80 & 6.00 & 24,00 & 1.16 & CMU263 & 67,30 & 3,80 & 16.80 & 0.89 & CMU627 & 128.00 & 6.10 & 35,60 & 0,66 \\
\hline CMA 190 & 32,00 & 4,40 & 13,70 & 0,44 & CMU268 & 57,00 & 3,90 & 15,40 & 0,62 & CMU628 & 129,41 & 5,00 & 23,00 & 0,86 \\
\hline CMA191 & 17,50 & 2,90 & 11,50 & 0,62 & CMU300 & 39,00 & 4,13 & 14,00 & 1,06 & CMU629 & 110,10 & 8,20 & 30,80 & 0,70 \\
\hline CIR196 & 99,20 & 6,00 & 25,00 & 1,12 & CMU 375 & 57,60 & 4,60 & 24,70 & 0,87 & CMU631 & 119,37 & 5,00 & 28,00 & 1,00 \\
\hline CIR201 & 52,20 & 5,50 & 15,40 & 0,79 & CMU376 & 57,00 & 5,97 & 24,30 & 0,13 & CMA639 & 89,70 & 3,40 & 21,30 & 0,71 \\
\hline CIR202 & 13,30 & 3,40 & 16,00 & 0,89 & CMU377 & 53,40 & 4,30 & 12,20 & 0,94 & CMU687 & 14,60 & 1,60 & 3,80 & 0,35 \\
\hline CIR203 & 52,50 & 2,70 & 17,00 & 0,54 & CMU378 & 56,10 & 5,50 & 22,20 & 0,41 & CMU690 & 90,30 & 4,40 & 28,20 & 0,51 \\
\hline CIR212 & 82,40 & 7,40 & 31,90 & 0,32 & CMU379 & 52,60 & 4,10 & 14,90 & 0,37 & CMU691 & 105,10 & 5,10 & 44,41 & 0,60 \\
\hline CIR213 & 65,10 & 2,90 & 17,70 & 0,40 & CMU380 & 75,50 & 6,00 & 27,00 & 1,06 & CMU692 & 115,30 & 7,60 & 38,60 & 0,38 \\
\hline CIR215 & 95,60 & 5,00 & 20,00 & 1,51 & CMU381 & 55,30 & 6,00 & 20,00 & 1,47 & CMU693 & 60,10 & 4,40 & 22,10 & 0,27 \\
\hline CIR217 & 82,10 & 4,00 & 25,00 & 1,14 & CMU382 & 33,40 & 3,90 & 11,40 & 0,25 & CMU696 & 39,70 & 2,60 & 9,12 & 0,46 \\
\hline CIR220 & 97,80 & 7,50 & 34,50 & $\begin{array}{l}0,44 \\
0,44\end{array}$ & CMU383 & 55,80 & 6,60 & 24,80 & 0,44 & CMU697 & 48,80 & 4,80 & $\begin{array}{r}19,20 \\
19,20\end{array}$ & $\begin{array}{l}0,40 \\
0,35\end{array}$ \\
\hline CMA222 & 143,00 & 8,77 & 41,00 & 1,10 & CMU384 & 64,90 & 5,50 & 22,20 & 0,24 & CMU698 & 36,70 & 4,30 & 23,10 & 0,75 \\
\hline CMA223 & 89,40 & 8,00 & 24,00 & 1,30 & CMU385 & 135,70 & 6,00 & 35,00 & 1,33 & CMU703 & 77,50 & 4,70 & 26,10 & 0,33 \\
\hline CMA224 & 130,88 & 10,00 & 48,00 & 1,04 & CMU387 & 39,40 & 6,00 & 16,30 & 0,84 & CMU706 & 63,40 & 3,20 & 17,50 & 0,65 \\
\hline CMA225 & 153,63 & 11,00 & 54,00 & 1,28 & CMU388 & 63,10 & 6,00 & 20,00 & 1,02 & CMU707 & 42,60 & 4,60 & 16,20 & 0,27 \\
\hline CMA227 & 173,31 & 10,00 & 54,00 & 1,54 & $\begin{array}{l}\text { CMU389 } \\
\text { ? }\end{array}$ & 61,70 & 4,00 & 15,00 & 1,53 & CMU708 & 44,30 & 2,90 & 14,40 & 0,74 \\
\hline CMA228 & 147,76 & 11,00 & 54,00 & 1,17 & CMU390 & 34,80 & 3,50 & 9,90 & 0,80 & CMU711 & 63,70 & 4,10 & 16,30 & 0,48 \\
\hline CMA229 & 124,40 & 8,40 & 31,10 & 1,20 & CMU391 & 67,50 & 6,20 & 20,50 & 0,35 & CMU714 & 84,10 & 5,50 & 31,20 & 0,40 \\
\hline CMA242 & 99,40 & 14,40 & 32,90 & 0,60 & CMU393 & 61,80 & 6,70 & 24,80 & 0,27 & CMU717 & 103,30 & 6,10 & 31,60 & 0,37 \\
\hline CMA247 & 157,20 & 14,00 & 85,00 & 1,53 & CMU394 & 47,70 & 3,60 & 18,50 & 0,44 & CMU718 & 58,70 & 4,10 & 19,80 & 0,32 \\
\hline CMA274 & 130,50 & 10,00 & 42,00 & 1,16 & CMU396 & 50,30 & 4,60 & 17,40 & 0,61 & CMU719 & 77,60 & 3,10 & 11,40 & 0,34 \\
\hline CMA276 & 137,14 & 11,00 & 48,00 & 1,17 & CMU397 & 61,10 & 6,32 & 26,30 & 0,95 & CMU722 & 23,90 & 4,40 & 13,60 & 1,10 \\
\hline CMA280 & $\begin{array}{r}84,40 \\
84\end{array}$ & 8,40 & $\begin{array}{l}4,000 \\
22,60\end{array}$ & $\begin{array}{l}1,17 \\
0,54\end{array}$ & CMU399 & 41,00 & $\begin{array}{l}0,52 \\
7,50\end{array}$ & 22,30 & 0,17 & CMU723 & $\begin{array}{r}102,80 \\
102,8\end{array}$ & $\begin{array}{l}4,40 \\
5,10\end{array}$ & $\begin{array}{l}1,00 \\
39,30\end{array}$ & $\begin{array}{l}1,10 \\
0,55\end{array}$ \\
\hline CMA285 & 116,70 & 5,20 & 24,40 & 0,48 & CMU501 & 66,50 & 4,50 & 23,90 & 0,33 & CMU725 & 79,80 & 4,30 & 24,40 & 0,66 \\
\hline CMA286 & 32,20 & 4,20 & 14,80 & 0,55 & CMU502 & 54,60 & 2,80 & 10,60 & 1,00 & CMU726 & 41,50 & 3,80 & 11,30 & 0,49 \\
\hline CMA287 & 109,30 & 8,50 & 31,20 & 0,26 & CMU503 & 35,90 & 3,50 & 13,10 & 0,53 & CMU798 & 51,80 & 4,50 & 18,00 & 0,47 \\
\hline CMA347 & 84,00 & 4,80 & 17,50 & 0,78 & CMU504 & 30,00 & 2,90 & 9,80 & 0,60 & CMU801 & 63,60 & 5,20 & 20,60 & 0,25 \\
\hline CMA348 & 112,10 & 5,80 & 32,20 & 0,44 & CMU505 & 65,50 & 2,88 & 11,00 & 0,98 & CMA846 & 60,40 & 10,10 & 45,40 & 1,07 \\
\hline CMA349 & 86,80 & 6,30 & 28,10 & 0,57 & CMU601 & 97,60 & 4,80 & 23,20 & 1,03 & CMA850 & 63,20 & 6,80 & 29,20 & 1,62 \\
\hline CMA350 & 99,70 & 7,50 & 27,40 & 0,77 & CMU605 & 84,90 & 5,00 & 19,00 & 1,17 & CMU860 & 55,40 & 3,60 & 17,70 & 0,16 \\
\hline CMA351 & 35,80 & 3,90 & 11,80 & 0,43 & CMU607 & 58,00 & 3,00 & 13,00 & 0,85 & CMU861 & 69,60 & 4,40 & 24,90 & 0,61 \\
\hline CMA358 & 74,00 & 7,00 & 21,30 & 1,28 & CMU608 & 121,00 & 7,00 & 33,00 & 2,04 & CMU862 & 82,17 & 5,60 & 21,20 & 0,72 \\
\hline CMA366 & 115,70 & 7,40 & 30,90 & 0,57 & CMU609 & 126,00 & 3,75 & 24,00 & 1,28 & CMU868 & 45,40 & 3,00 & 15,50 & 0,23 \\
\hline CMA367 & 52,50 & 13,70 & 12,80 & 0,24 & CMU610 & 89,12 & 4,00 & 16,10 & 1,26 & CMU871 & 119,30 & 3,60 & 28,60 & 1,55 \\
\hline CMA368 & 72,10 & $\begin{array}{r}1,70 \\
9,60\end{array}$ & $\begin{array}{l}1,2,00 \\
27,30\end{array}$ & $\begin{array}{l}0,24 \\
0,59\end{array}$ & CMU611 & $\begin{array}{r}89,12 \\
133,30\end{array}$ & $\begin{array}{r}4,00 \\
13,00\end{array}$ & $\begin{array}{l}10,10 \\
47,00\end{array}$ & $\begin{array}{l}1,20 \\
1,22\end{array}$ & CMU874 & $\begin{array}{r}83,40 \\
83,40\end{array}$ & $\begin{array}{l}5,00 \\
5,70\end{array}$ & $\begin{array}{l}28,00 \\
28,10\end{array}$ & 0,41 \\
\hline CMA369 & 66,30 & 8,20 & 31,60 & 0,62 & CMU612 & 92,00 & 8,00 & 26,00 & 1,62 & CMU877 & 101,20 & 4,60 & 22,40 & 0,65 \\
\hline CMA370 & 58,40 & 7,70 & 29,30 & 0,85 & CMU613 & 117,00 & 10,00 & 38,00 & 1,28 & CMU879 & 48,20 & 3,90 & 13,70 & 0,29 \\
\hline CMA371 & 96,30 & 9,10 & 42,30 & 0,80 & CMU614 & 76,60 & 5,70 & 23,30 & 0,91 & CMU880 & 79,50 & 7,00 & 30,60 & 0,39 \\
\hline CMA372 & 38,30 & 4,10 & 9,60 & 1,05 & CMU615 & 98,50 & 7,00 & 18,00 & 1,04 & CMU881 & 33,30 & 2,80 & 8,70 & 0,17 \\
\hline CMA374 & $\begin{array}{l}82,70 \\
82,70\end{array}$ & $\begin{array}{l}4,10 \\
9,20\end{array}$ & $\begin{array}{l}3,00 \\
39,90\end{array}$ & $\begin{array}{l}1,03 \\
0,59\end{array}$ & CMU616 & $\begin{array}{l}90,00 \\
157,20\end{array}$ & 5,00 & $\begin{array}{l}10,00 \\
20,00\end{array}$ & $\begin{array}{l}1,04 \\
1,00\end{array}$ & CMU882 & $\begin{array}{l}50,00 \\
68,80\end{array}$ & $\begin{array}{l}2,00 \\
2,60\end{array}$ & $\begin{array}{r}0,70 \\
10,50\end{array}$ & $\begin{array}{l}0,11 \\
0,91\end{array}$ \\
\hline CMA426 & 98,90 & 7,90 & 26,30 & 1,30 & CMU617 & 89,80 & 4,00 & 24,00 & 1,13 & CMU886 & 42,60 & 3,80 & 14,40 & 0,23 \\
\hline CMA427 & 82,10 & 4,70 & 21,30 & 0,56 & CMU618 & 64,10 & 3,12 & 10,19 & 1,10 & CMU 888 & 57,80 & 5,40 & 19,00 & 0,49 \\
\hline CMA431 & 112,50 & 10,30 & 46,30 & 0,50 & CMU619 & 147,80 & 5,00 & 41,00 & 1,00 & CMU897 & 70,70 & 5,60 & 18,70 & 0,44 \\
\hline CMA433 & 107,50 & 7,00 & 36,10 & 0,47 & CMU620 & 133,60 & 7,00 & 27,00 & 1,00 & CMU900 & 82,00 & 3,40 & 19,80 & 0,39 \\
\hline CMA436 & 80,80 & 5,40 & 21,70 & 0,33 & CMU621 & 83,10 & 7,50 & 22,10 & 0,70 & CIR849 & 75,10 & 5,50 & 55,50 & 0,53 \\
\hline CMA437 & $\begin{array}{l}0.00 \\
89,40\end{array}$ & $\begin{array}{l}5,40 \\
5,10\end{array}$ & 20,40 & 0,46 & CMU622 & $\begin{array}{l}83,10 \\
74,00\end{array}$ & $\begin{array}{l}7,50 \\
6,50\end{array}$ & $\begin{array}{l}2,10 \\
18,90\end{array}$ & $\begin{array}{l}0,10 \\
0,88\end{array}$ & $\begin{array}{l}\text { CIR903 } \\
\text { (1) }\end{array}$ & 49,75 & 6,80 & $\begin{array}{l}27,90 \\
2\end{array}$ & $\begin{array}{l}0,48 \\
0,48\end{array}$ \\
\hline CMA514 & 76,00 & 3,60 & 16,70 & 0,54 & CMU623 & 57,34 & 7,00 & 20,00 & 1,03 & & & & & \\
\hline CMU174 & 64,80 & 7,70 & 18,30 & 0,29 & CMU624 & 73,60 & 7,00 & 27,00 & 1,00 & & & & & \\
\hline
\end{tabular}

${ }^{(1)} \mathrm{CMU}$ : Clones procedentes de Maués; CMA: Clones procedentes de Manaus; CIR: Clones procedentes de Iranduba. 
rioridade de seus híbridos, conforme Ferreira (1993), em milho. Por outro lado, Oliveira (1995) relata que a média de uma população segregante depende da freqüência dos locos fixados com alelos favoráveis, e da freqüência de locos em heterozigose. Quando os genitores utilizados são adaptados, a freqüência de locos favoráveis é alta.

Os clones utilizados são materiais com alta freqüência de locos em heterozigose, porém não se pode afirmar que exista alta frequiência de locos com alelos favoráveis fixados, pois a clonagem foi realizada em plantas-matrizes cuja população não tinha sido melhorada, e que a expressão fenotípica poderia estar sendo grandemente influenciada pelo ambiente. Entretanto, a formação de populações segregantes, a partir de cruzamentos biparentais ou múltiplos, entre os melhores clones de cada grupo, aumentaria a probabilidade de surgimento de combinações híbridas superiores.

Em relação à propagação vegetativa, principalmente os clones geneticamente mais próximos, como é o caso dos clones CMU384 e CMU801, poderiam ser utilizados para formação de uma população com desenvolvimento vegetativo uniforme e de base ge-

Tabela 2. Resumo das estimativas das distâncias genéticas, com base na distância euclidiana média padronizada, entre 148 clones de guaranazeiro, para os caracteres comprimento do ramo principal (CRP), em $\mathrm{cm}$, número de ramos (NR) e número de folhas (NF), aos 12 meses de idade, e produção de sementes torradas (PROD), em kg/planta

\begin{tabular}{lrr}
\hline Distância genética & Estimativa & \multicolumn{1}{c}{ Clone } \\
\hline Máxima & 5,06660 & CMA247 e CMU687 \\
Mínima & 0,09503 & CMU384 e CMU801 \\
Soma das estimativas & 13657,51855 & \\
Soma de quadrados das & 21756,05078 & \\
estimativas & & \\
Média das estimativas & 1,25552 & \\
\hline
\end{tabular}

Tabela 3. Grupos de progenitores estabelecidos pelo método de Tocher, com base na dissimilaridade expressa pela distância euclidiana média padronizada.

\begin{tabular}{lc}
\hline Grupo & Progenitores \\
\hline 1 & Demais clones \\
2 & CMA225, CMA228, CMA276, CMA224, CMA274, \\
& CMA222, CMU613, CMU611, CMA227, CMA431, \\
& CMU385, CMU619, CMA846, CMU812 e CMU608 \\
3 & CMU616 e CMU871 \\
4 & CMA242 e CMA367 \\
5 & CIR849 \\
6 & CMU687 \\
7 & CMA247 \\
\hline
\end{tabular}

nética não restrita a uma única fonte, ou seja um "pool" clonal, para plantio em condições comerciais.

Existe variabilidade genética entre os grupos formados pelo método de Tocher, nas quatro características consideradas na avaliação (Tabela 4). Portanto, existe possibilidade de ganhos com a seleção de progênies provenientes de cruzamentos entre clones de grupos distintos.

O dendrograma formado pelo método do vizinho mais próximo, a partir das distâncias genéticas entre os grupos, permite visualizar que os grupos 1,4 e 5 são muito próximos, e podem ser considerados como um único grupo (Figura 1).

Tabela 4. Resumo das análises de variância dos caracteres comprimento do ramo principal (CRP), em $\mathrm{cm}$, número de ramos (NR), e número de folhas (NF), aos 12 meses de idade, e produção de sementes torradas (PROD), em kg/planta.

\begin{tabular}{lccccc}
\hline Fonte de variação & \multirow{2}{*}{ GL } & \multicolumn{4}{c}{ Quadrados médios } \\
\cline { 3 - 6 } & & CRP & NR & NF & PROD \\
\hline Entre grupos & 6 & $9,6188^{* *}$ & $12,3780^{* *}$ & $14,1897^{* *}$ & $6,4708^{* *}$ \\
Dentro de grupos & 141 & 0,6332 & 0,5158 & 0,4387 & 0,7672 \\
\hline CV $(\%)$ & & 33,27 & 30,18 & 31,29 & 45,59 \\
\hline
\end{tabular}

**Significativo a $1 \%$ de probabilidade, pelo teste $\mathrm{F}$.



Figura 1. Dendrograma de distâncias genéticas entre grupos de clones de guaranazeiro obtido pelo método do vizinho mais próximo. Grupo 1: Demais clones; Grupo 2 : CMA225, CMA228, CMA276, CMA224, CMA274, CMA222, CMU613, CMU6111, CMA227, CMA431, CMU385, CMU619, CMA846, CMU812 e CMU608; Grupo 3: CMU616 e CMU871; Grupo 4: CMA242 e CMA367; Grupo 5: CIR849; Grupo 6: CMU687; Grupo 7 : CMA247. 


\section{Conclusões}

1. A divergência genética dos clones atualmente em uso no programa de melhoramento genético do guaranazeiro da Embrapa-Centro de Pesquisa Agroflorestal da Amazônia Ocidental não é grande.

2. A formação de populações segregantes, a partir de cruzamentos biparentais ou múltiplos, entre os clones mais produtivos de cada grupo, aumenta a probabilidade de surgimento de combinações híbridas superiores.

3. Os clones CMU384 e CMU801 são os mais próximos, geneticamente, e podem ser utilizados na formação de uma população com desenvolvimento vegetativo uniforme para uso em plantios comerciais.

\section{Referências}

BOLETIM AGROMETEOROLÓGICO. Manaus : Embrapa-CPAA, 1998. 23 p.

CAVALCANTE, P. B. O guaraná (Paullinia cupana var. sorbilis) em estado provavelmente espontâneo, no planalto de Santarém, Pará. Boletim do Museu Paraense Emílio Goeldi, Belém, n. 26, p. 1-5, jan. 1967.

CRUZ, C. D. Aplicação de algumas técnicas multivariadas no melhoramento de plantas. Piracicaba : ESALQ, 1990. 188 p. Tese de Doutorado.

CRUZ, C. D. Programa GENES: aplicativo computacional em genética e estatística. Viçosa : UFV, 1997. $442 \mathrm{p}$
CRUZ, C. D.; REGAZZI,A. J. Modelos biométricos aplicados ao melhoramento genético. 2. ed. Viçosa : UFV, 1997. $390 \mathrm{p}$.

DUCKE, A. Diversidade dos guaranás. Rodriguésia, Rio de Janeiro, v. 3, n. 10, p. 155-156, 1937

FERREIRA, D. F. Métodos de avaliação da divergência genética em milho e suas relações com os cruzamentos dialélicos. Lavras : UFLA, 1993. 72 p. Dissertação de Mestrado.

MACHADO, C. F. Procedimentos para a escolha de genitores de feijão. Lavras : UFLA, 1999. 118 p. Dissertação de Mestrado.

MURTY, B. R.; ARUNACHALAM, V. The nature of genetic divergence in relation to breeding system in crop plants. Indian Journal of Genetics, New Delhi, v. 26, p. $188-189,1966$

NASCIMENTO FILHO, F. J. do; CRUZ, C. D.; GARCIA, T. B. Divergência genética em plantas jovens de guaranazeiro e possibilidades de melhoramento. Pesquisa Agropecuária Brasileira, Brasília, v. 27, n. 12, p. 15711577, dez. 1992

OLIVEIRA, L. B. Alternativas na escolha dos parentais em um programa de melhoramento do feijoeiro. Lavras : UFLA, 1995. 60 p. Dissertação de Mestrado.

UPADHYAY, M. K.; MURTY, B. R. Genetic divergence in relation to geographical distribution in pearl millet. Indian Journal of Genetics, New Delhi, v. 30, p. $704-$ 715,1970 . 